\title{
What Business Schools Can Learn from the Medical Profession
}

Christof Schmitz,

Peter Berchtold

Co-Leiter college M
Business Schools wie Harvard fungieren als Leuchttürme des Managements. Wer einen Harvard-MBA hat, darf in Anspruch nehmen, top of the line $\mathrm{zu}$ sein. Das galt lange Zeit unbestritten. Mit der Krise von 2008 , die viele wirtschaftliche, soziale wie auch ethische Fragen aufwarf, begann eine intensive Diskussion, inwieweit die Art und Weise und die Inhalte der Management-Lehre adäquat für die Praxis sind. Reform stand an. Als Nitin Nohira, der Dekan von Harvard, jüngst das neue Modell dieser prominenten Managementschule präsentierte, stellte er das Management-Lernen in eine interessante Beziehung zur medizinischen Profession: Statt wie bisher abstrakte Fälle zu bearbeiten, werden die Teilnehmenden nun realen Situationen in Unternehmen ausgesetzt und dabei von den Harvard-Dozenten betreut [1]. Als Modell dafür dient die Medizin und die Art und Weise, wie Ärzte ausgebildet und angeleitet werden. Assistentinnen und Assistenten behandeln Patienten und werden dabei von erfahrenen Ärzten supervidiert. Diese Art von Lernen wird von Harvard nun als Leitform betrachtet, um praxisorientiertes Lernen zu ermöglichen. Das ist bemerkenswert, sowohl für die Medizin, die sich freuen kann (wieder einmal) als Vorbild für Management ein Interpretieren, was der Fall ist, und immer wieder kreatives Überlegen, was wirkungsorientiertes Handeln sein kann.

Ebenso wenig wie komplexe Patienten ausreichend durch Guidelines behandelt werden können, ordnet sich die komplexe Führungsrealität ausreichend der Logik auch noch so elaborierter Instrumente unter. Führung ist kontext- und erfahrungsgebunden und verlangt Wissen genauso wie Haltung und Skills. Das ist es, was Führung anspruchsvoll, aber auch spannend macht. Das gilt es zu berücksichtigen, wenn man (seine) Führung weiterentwickeln will. Und das ist das Argument, das Harvard nun anführt: Lernen geschieht in der Praxis und soll hier gefördert, angereichert und reflektiert werden.

Angehende Ärztinnen und Ärzte lernen in der klinischen Arbeit nicht nur, was sie mit Patienten in bestimmten Fällen medizinisch machen sollen. Sie lernen gleichzeitig, wie sie mit Patienten umgehen sollen: wie kommunizieren, wie schwierige Diagnosen mitteilen, wie für Compliance sorgen, wie ethisch entscheiden usw. Exakt das Gleiche gilt für Führung. Nicht nur Wissen ist relevant - z. B. welche Aufgaben Führung umfasst und welche Instrumente es gibt sondern ebenso das Wie: Wie gestalte ich die Bezie-

\section{Statt wie bisher abstrakte Fälle zu bearbeiten, werden die Harvard- Studierenden jetzt realen Situationen in Unternehmen ausgesetzt - als Modell dient die Medizin.}

1 Nohira N. What Business Schools Can Learn from the Medical Profession. HBR. January-February 2012;38.

\section{Korrespondenz:}

Dr. Christof Schmitz

college M

Freiburgstrasse 41

CH-3010 Bern

christof.schmitz[at]college-m.ch zu fungieren, als auch für das Management bzw. für die Frage, was adäquates Führungslernen ist. Wird doch zugestanden, dass Management eine Praxisform ist, die tief eingebettet ist in konkrete, soziale Kontexte.

\section{Nicht Guideline-tauglich}

Was zählt, ist die Praxis und nicht die Theorie. Wer Anatomie gelernt hat, weiss noch nicht, was er mit einem konkreten Patienten tun soll, der über Rückenschmerzen klagt. Wer Führungskonzepte gelernt hat, weiss noch nicht, was sie tun soll, wenn konkrete Mitarbeiter über Probleme klagen. (Auch) Führung ist eben mehr als die Anwendung irgendwelcher Tools oder Checklisten. Führung ist waches Wahrnehmen einer mannigfaltigen Wirklichkeit, hungen zu meinen Mitarbeitenden, wie gehe ich mit Schwierigkeiten um, wie bleibe ich fair, wie entscheide ich in komplexen Fällen auch bei unzureichender Faktenlage - und mit welchen Wirkungen?

\section{Von der Medizin lernen}

Die Medizin ist eine Profession, die sich nicht in der blinden Anwendung von Wissen erschöpft, sondern ihre Spezifität aus dem Intervenieren in Krisen somato-psycho-sozialer Integrität von Menschen bezieht. Das Umgehen mit dieser Krisenhaftigkeit, das erst macht sie zur Profession. In solchen Krisen auf der Höhe des Wissens zu sein und bezogen und verantwortlich $\mathrm{zu}$ handeln, das ist die professionelle Aufgabe; «do no harm ...». Management sieht sich nicht nur krisenhaften, sondern auch gestalte- 


\section{Nachdiplomkurs «Wirksam managen im Gesundheitssystem» 2012/13}

Management- und Führungskompetenz muss auch im Kerngeschäft vertreten sein. Ein anspruchsvolles medizinisches Kerngeschäft ist dort ebenso zu führen, wie vielfältige andere Interessen und Erwartungen zu berücksichtigen und einzubinden sind. Ärztinnen und Ärzte bewegen sich dabei in komplexen Netzwerken, die aktuell starken Veränderungen unterworfen sind. Darin zu guten Lösungen beizutragen, das macht Management im Gesundheitswesen anspruchsvoll und will gelernt sein.

\section{Der Nachdiplomkurs «Wirksam managen»}

- vermittelt einen auf die Besonderheiten des Managens in Organisationen des Gesundheitssystems bezogenen Ansatz.

- vermittelt Wissen, Haltungen und Fähigkeiten für erfolgreiches Handeln in den komplexen Netzwerken von Organisationen des Gesundheitswesens.

- bietet Orientierung und Übersicht und verfolgt einen stärkenorientierten Zugang zur Führungsthematik.

- vermittelt Wissen über die aktuellen Entwicklungen des Gesundheitswesens.

- betreibt eine konsequente Verschränkung der vermittelten Themen mit der jeweils individuellen Handlungspraxis.

- baut konkrete Feedback-Schleifen ein und stärkt dadurch den Praxisbezug und die Effektivität des Programms.

«Wirksam managen» gliedert sich in 6 Module von insgesamt 17 Tagen. Die einzelnen Module bilden zusammen einen kohärenten Lernprozess. Die Effektivität des Programms wird durch Feedback-Schleifen zwischen den Teilnehmenden und ihren Kontexten zusätzlich gesteigert. Wichtig ist auch eine Lernkultur, die Freude und Neugier befördert und Austausch und Anregung durch andere unterstützt. Der didaktische Bogen reicht von Theorie und Evidenzorientierung bis zum erfahrungsbasierten Lernen im Rahmen der Bearbeitung konkreter Beispiele.

Der Nachdiplomkurs wird mit einem Zertifikat des college $\mathrm{M}$ abgeschlossen und ist FMH approved (8 Credits pro Modul).

Modul 1: Managen (Montag, 20.8.2012 bis Mittwoch, 22. 8. 2012)

- Zentrale Management- und Führungskonzepte heute

- Die Besonderheit von Spitälern und anderen Organisationen im Gesundheitswesen und ihre Konsequenzen fürs Management

- Orientierung im Dschungel des Führens: die Leadership Map

Zwischen den Modulen: Stärkenorientiertes Feedback einholen

Modul 2: Führen (Montag, 1. 10. 2012 bis Mittwoch, 3. 10. 2012)

- Mit anderen zusammenarbeiten: von High-Performance lernen

- Professionelle Kooperation und Führung: Teams, Netzwerke, Hierarchien

Führung konkret (Assessment und Feedback)

Zwischen den Modulen: Peergestützt Feedbacks einholen

Modul 3: Intervenieren (Mittwoch, 14. 11. 2012 bis Freitag, 16. 11. 2012)

- Medizin und Ökonomie - Wirkungen und Nebenwirkungen

- Den Prozess der Führung konkretisieren und durchspielen

Konkrete Beispiele bearbeiten

Modul 4: Wirken (Montag, 7. 1. 2013 bis Mittwoch, 9. 1. 2013)

- Kommunikation als Medium von Führung

- Wie man Wirkung erzielt

Modul 5: Steuern (Montag, 4. 3. 2013 bis Mittwoch 6. 3. 2013)

- Selbstmanagement: Führ Dich selbst, sonst führt dich keiner

- Strategisches Management im Gesundheitssystem

- Die Organisation gestalten und entwickeln

Modul 6: Integrieren (Donnerstag, 11.4.2013 bis Freitag, 12.4.2013)

- Die vielfältigen Facetten der Führung integrieren

- Rückblick und Ausblick

Verantwortliche Programmleiter: PD Dr. med. Peter Berchtold und Dr. Christof Schmitz, Co-Leiter college-M

Ort: college M, Freiburgstrasse 41, 3010 Bern

Preis: Die Kurskosten betragen 7950 Franken und beinhalten alle Programmaktivitäten und Unterlagen.

Informationen und Anmeldung: Detaillierte Informationen finden Sie unter www.college-m.ch E-Mail: weiter.bildung[at]college-m.ch, Tel. 0316323026.

"College M vermittelt im Bereich «Management und Führung im Spital» für die Ärzte sowohl gutverständliche theoretische Grundlagen als auch sehr praxisorientierte Übungsanlagen. Der Unterricht in der Gruppe ist innovativ und kreativ gestaltet, aber dennoch nüchtern fokussiert auf die relevanten Alltagsprobleme. Fazit: eine gute Möglichkeit für Kaderärzte, ihr Rüstzeug in Management und Führung zu erarbeiten und weiterzuentwickeln.» (Prof. Dr. med. Thomas J. Neuhaus, Chefarzt Pädiatrie Kinderspital Luzern) rischen Aufgaben gegenüber. Gleichwohl gilt auch hier: Es geht um Wahrnehmen, Verstehen und verantwortliches Intervenieren in komplexe Wirklichkeiten. Wirklichkeiten, die nicht-trivialer, nichttechnischer Natur sind. Die dafür nötige Art von Intervenieren lernt man nur durch konkrete Erfahrungen, nicht durch Wissensaneignung allein. Erfahrungen sind umfangreicher, sie integrieren Denken mit Fühlen und Handeln, wie uns die Neurobiologie gelernt hat. Darum ist kein Betriebswirtschaftsstudent per se eine gute Führungskraft und kein MBA-Absolvent von vornherein ein begnadeter Manager. Erst die Kombination von Mensch und Praxis in der konkreten Situation entscheidet.

Die Harvard-Geschichte ist nicht das erste Mal in den letzten Jahren, dass Medizin zum Vorbild für Management gereicht. Insbesondere der Erfolg der evidenzbasierten Medizin bedeutete für den Managementbereich einen Wake up call, der zur Einsicht führte, dass in der Managementpraxis viel zu wenig nach der Evidenz zur Wirksamkeit von Managementkonzepten und Methoden gefragt wird. Der Ruf nach evidence-based management wurde laut. Dabei beschäftigte nicht nur das rigorose Überprüfen von Wissen und Daten an sich, sondern auch, wie solches in medizinische Entscheidungsprozesse eingebunden wird. Was das Management fasziniert, ist eben diese Spannweite, die von Rigorosität in Entscheidungsfindung und der Nutzung wissenschaftlichen Wissens bis zur konkreten, immer auch diffusen Praxis der Behandlung eines einzelnen Patienten reicht. Und die Frage, wie hohe professionelle Ansprüche und anspruchsvolle, «ungenaue» Praxis miteinander zu kombinieren sind.

\section{Anforderungen an Führungslernen}

Worauf die Harvard-Reform hinausläuft, ist das Ernstnehmen der Praxisgebundenheit von Führung und Management. Führungsentwicklung kann weder rein kognitives Lernen irgendwelcher Theorien und Konzepte bedeuten noch (kontext-) blindes Anwenden von Führungswissen oder Instrumenten meinen. Führungslernen beinhaltet selbstverständlich die Aneignung von Konzepten und Modellen - Wissen - und muss ebenso selbstverständlich den Bezug zur eigenen konkreten Führungspraxis und zur eigenen Person implizieren. Effektives Führungslernen integriert immer Wissen und Handeln. Der reflexive Bezug zur eigenen Person im konkreten Kontext ist dabei unabdingbar. Dazu kommt noch die Gelegenheit der Erprobung neuer Verhaltensweisen. Nur wer neue Erfahrungen macht, lernt (besser) führen - alles andere bleibt buchstäblich «graue Theorie». Let's do it. 